\title{
Approach to exact solutions of cosmological perturbations: Tachyon field inflation
}

\author{
Ramón Herrera* and Roberto G. Péredt \\ Instituto de Física, Pontificia Universidad Católica \\ de Valparaíso, Casilla 4059, Valparaíso, Chile.
}

(Dated: July 23, 2018)

\begin{abstract}
An inflationary universe scenario in the context of tachyon field is studied. This study is done from an ansantz for the effective potential of cosmological perturbations $U(\eta)$. We describe in great detail the analytical solutions of the scalar and tensor perturbations for two different ansantz in the effective potential of cosmological perturbations; the Easther's model and an effective potential similar to power law inflation. Also we find from the background equations that the effective tachyonic potential $V(\varphi)$, in both models satisfy the properties of a tachyonic potential. We consider the recent data from the Planck data to constrain the parameters in our effective potential of cosmological perturbations.
\end{abstract}

PACS numbers: $98.80 . \mathrm{Cq}$

*Electronic address: ramon.herrera@ucv.cl

$\dagger$ Electronic address: genaro.perez@gmail.com 


\section{INTRODUCTION}

It is well known that the inflationary scenario provides an approach for solving the problems of the standard big bang model[1, 2]. However, the inflationary universe model can offer a graceful mechanism to explain the large-scale structure [3], and likewise the observed anisotropy of the cosmic microwave background (CMB) radiation [4, 5].

On the other hand, implications of string/M-theory to Friedmann-Roberson-Walker (FRW) models have great attention, and in particular then we considered a tachyonic field associated with unstable D-branes[6]. However, an important characteristic of the tachyon field is that this field might be responsible of the inflationary scenario at early times of the universe [7, 8], and also of the dark energy [9, 10]. Nevertheless, the tachyonic field could at the same time produce an inflationary scenario, and after generate an epoch dominated by a non-relativistic fluid, which could add to the dark matter[11]. Different effective tachyonic potentials have been considered in the literature, and in particular an exponential potential constructed from the string theory [12], modifications of this [13] and among others. The characteristic of the effective tachyonic potential $V(\varphi)$, where $\varphi$ denotes a homogeneous tachyon field are: $\partial V(\varphi>0) / \partial \varphi<0$, the potential $V$ has a global maximum at $\varphi \sim 0$, and $V(|\varphi| \rightarrow \infty) \rightarrow 0$. For a review of tachyon inflation, see Refs. 14, 15]

In the context of the cosmological perturbations, the power spectrums of the scalar perturbations, and tensor perturbations are originated from quantum fluctuations of the vacuum in an expanding universe[16]. It is well known that these fluctuations have considerable amplitudes in the very early universe (Planckian length), and during the expansion of the universe in the inflationary epoch, these fluctuations are extended with amplitudes beyond of galactic scales. In relation to exact solutions of the cosmological perturbations and the equations of motion (background space times) for a standard scalar field, these can be calculated exactly in the Sitter inflationary universe, in which a constant potential is found from the equations of motion of background[1], and also exact results for the spectrums of the cosmological perturbations[16]. Also, in the expansion power law or power law inflation, we can obtained exact results from the background equations (exponential potential)[17], and exact calculations for the cosmological perturbations, see Ref.[18]. Similarly, exact results can be obtained in the Easther's model, where the effective potential of cosmological perturbations is $U=0[19]$. However, this model has an spectral index $n_{S} \equiv 3$ (blue tilt), which 
is significantly different from the observational data. Generalizations from these spectrums, were obtained in Ref.[20], however the background solutions was obtained numerically. Also, some approximations to the cosmological perturbations are studied in Refs. [21, 22]

In the following, we explore an approach to exact results in a tachyonic inflationary model. We propose this possibility in the context of an ansatz for the effective potential of cosmological perturbations, and we describe how this ansatz influences the dynamics of our models, and the solutions of the cosmological perturbations. We follow a similar procedure described in the Easther's model, and after we study an effective potential of cosmological perturbations studied in Ref.[20].

The outline of the paper goes as follow: In the sections II y III we present a short description of the background equations and the cosmological perturbations for the tachyon field. In the section IV we study the Easther's model. In the section V we give explicit expressions for the scalar and tensor spectrums, considering a specific ansatz for the effective potential of cosmological perturbations. At the end, section VI exhibits our conclusions. We chose units so that $c=\hbar=1$.

\section{BACKGROUND MODEL: TACHYON FIELD}

In this section we give a brief description of an inflationary model, considering that the tachyonic field drive inflation.

In the context of the Born-Infeld form, the effective action for tachyon could be described by [23]

$$
S=\int \sqrt{-g}\left(\frac{R}{2 \kappa}-V(\varphi) \sqrt{1+g^{\mu \nu} \partial_{\mu} \varphi \partial_{\nu} \varphi}\right) d^{4} x
$$

where the constant $\kappa$ is denoted by $\kappa=8 \pi G, R$ is the 4-dimensional Ricci scalar, derived from the metric tensor $g_{\mu \nu}$, and the field $\varphi$ is the tachyon scalar field with minimal coupling to gravity. Here, the effective scalar potential of the tachyon scalar field is denoted by $V(\varphi)$.

By taking the variation of action of Eq.(1) with respect to the two independent variables $g_{\mu \nu}$ and $\varphi$, it leads to two main dynamical equations. Let us introduce into action (11) the flat Friedmann-Lemaitre-Robertson-Walker (FLRW) metric, given by

$$
d s^{2}=d t^{2}-a^{2}(t) d x^{i} d x_{i}=a^{2}(\eta)\left[d \eta^{2}-d x^{i} d x_{i}\right]
$$

where $\eta$ is the conformal time defined as $d t=a d \eta$, the quantities $d x^{i} d x_{i}$ and $a(t)$ represent 
the flat three-surface and scale factor, respectively.

The energy-momentum tensor of tachyon field yields

$$
T_{\mu \nu}=\frac{V(\varphi)}{\sqrt{1+\varphi^{, c} \varphi_{, c}}}\left[-g_{\mu \nu}\left(1+\varphi^{, c} \varphi_{, c}\right)+\varphi_{, \mu} \varphi_{, \nu}\right],
$$

and admits to be cast into the form of a perfect fluid where $T_{\mu}^{\nu}=\operatorname{diag}(-\rho, p, p, p)$, in which the energy density $\rho$ and pressure $p$ are given by

$$
\rho=\frac{V(\varphi)}{\sqrt{1-\dot{\varphi}^{2}}}, \quad \text { and } \quad p=-V(\varphi) \sqrt{1-\dot{\varphi}^{2}},
$$

respectively. Here, the dots mean derivatives with respect to time $t$.

If the stress energy of the Universe is dominated for a spatially homogeneous tachyon field $\varphi(t)$, then the Friedmann equation, is given by

$$
H^{2}=\frac{\kappa}{3} \frac{V(\varphi)}{\sqrt{1-\dot{\varphi}^{2}}}
$$

and the Raychaudhuri equation in the case of the tachyonic matter yields

$$
\frac{\ddot{a}}{a}=H^{2}+\dot{H}=\frac{\kappa}{3} \frac{V(\varphi)}{\sqrt{1-\dot{\varphi}^{2}}}\left(1-\frac{3}{2} \dot{\varphi}^{2}\right),
$$

where $H$ is the Hubble parameter defined as $H \equiv \dot{a} / a$.

The inflationary scenario occurs when $\ddot{a}>0$, then from Eq.(15), we get $\dot{\varphi}^{2}<2 / 3$. Note that this condition for the velocity of the scalar tachyon field is different from the standard scalar field in which $\dot{\varphi}^{2}<V$.

The dynamical equation of motion of the tachyon field from Eq.(1) becomes

$$
\ddot{\varphi}+3 H \dot{\varphi}\left(1-\dot{\varphi}^{2}\right)+\frac{1}{V} \frac{d V}{d \varphi}\left(1-\dot{\varphi}^{2}\right)=0,
$$

which is equivalent to the conservation equation $\dot{\rho}+3 H(\rho+p)=0$.

Combining Eqs. (44) and (5), we get

$$
\dot{\varphi}^{2}=-\frac{2}{3} \frac{\dot{H}}{H^{2}}
$$

and the effective potential is given by

$$
V=\frac{3}{\kappa} H^{2} \sqrt{1+\frac{2}{3} \frac{\dot{H}}{H^{2}}} .
$$

Here, we have used Eqs.(44) and (17). 


\section{PERTURBATIONS}

In this section we will study the scalar and tensor perturbations for our models. We start with the most general metric perturbations

$$
d s^{2}=a^{-2}(\eta)\left[(1+A) d \eta^{2}-2 \partial_{i} B d x^{i} d \eta-\left[(1-2 R) \delta_{i j}+2 \partial_{i} \partial_{j} E\right] d x^{i} d x^{j}\right] .
$$

In this metric four amounts are needed to specify its general nature. These quantities are $A$, $B, R$ and $E$, are functions of the time and space coordinates and $\eta$ as before is the conformal time $\left(\eta=\int d t / a(t)\right)$.

It is well known that the scalar perturbations can be measured by the intrinsic curvature perturbation $\mathcal{R}$ of the comoving hypersurfaces, that in the case of the standard scalar field is given by $\mathcal{R}=-R-(H / \dot{\phi}) \delta \phi$. Here, $\phi$ is the standard scalar field and $\delta \phi$ represents the fluctuation of the scalar field. One important quantity to describe the perturbations is the gauge-invariant potential $u$, which is given by $u=a[\delta \phi+(\dot{\phi} / H) R] \equiv-z \mathcal{R}$, where the new variable $z$ in the case of the standard scalar field is defined as

$$
z \equiv a \dot{\phi} / H
$$

Here we note the quantities $\dot{\phi}$ and the Hubble parameter $H$ (or the scale factor) are determined by the background field equations.

On the other hand, the equations of motion of the linear scalar perturbation for the standard scalar field can be obtained from the action given by [16]

$$
S=\frac{1}{2} \int d \eta d^{3} x\left(u^{\prime 2}-\delta^{i j} \partial_{i} u \partial_{j} u+\frac{z^{\prime \prime}}{z} u^{2}\right),
$$

where a prime denotes differentiation with respect the conformal time $\eta$.

However, the action given by Eq.(11) is formally equivalent to the action for a scalar field with a time-dependent effective mass $m^{2}=-z^{\prime \prime} / z$ in flat space-time, and this equivalence implicates that one can take into account the quantum theory.

From the action (11), the equation for the Fourier modes $u_{k}$, for the standard scalar field can be written as

$$
u_{k}^{\prime \prime}+\left(k^{2}-\frac{z^{\prime \prime}}{z}\right) u_{k}=0
$$

where $k=|\boldsymbol{k}|$ is the modulus of the wavenumber. In this form, the Eq.(12) may be considered as a time independent Schrödinger equation given by $u_{k}^{\prime \prime}+\left(k^{2}-U\right) u_{k}=0$, where the 
effective potential is defined as $U=U(\eta)=z^{\prime \prime} / z$. From the Wronskian condition we have $u_{k}^{*} u_{k}^{\prime}-u_{k} u_{k}^{*^{\prime}}=-i$, that guarantees the commutation relations from the quantum theory. For a review of the cosmological perturbations of a standard scalar field, see Refs. [24, 25]

In the case of a tachyonic field, the calculation of the power spectrum can be obtained through a canonical variable, similarly to the standard scalar field. The variable $z$ to the case of a tachyon field can be written as [26 28]

$$
z=\frac{\sqrt{3} a \dot{\varphi}}{\left(1-\dot{\varphi}^{2}\right)^{1 / 2}},
$$

and the analogous equation to Eq.(12) for the Fourier modes $u_{k}$ in the case of a tachyonic fluid is given by [27]

$$
u_{k}^{\prime \prime}+\left(k^{2}\left(1-\dot{\varphi}^{2}\right)-U\right) u_{k}=0,
$$

where the term $\left(1-\dot{\varphi}^{2}\right)=c_{S}^{2}=\delta p / \delta \rho$, corresponds to the effective speed of sound, and $U$ as before corresponds to effective potential $U=U(\eta)=z^{\prime \prime} / z$. Here, we note that for a standard scalar field the effective speed of sound $c_{S}=1$.

The asymptotic limits for the equation of the Fourier modes $u_{k}$ given by Eq.(14) are: Firstly, we consider that in the limit when the conformal time $\eta \rightarrow-\infty$ corresponds to a small scale limit, where the modes are inside the horizon. Secondly, we take into account that in the limit when the conformal time $\eta \rightarrow 0$ represents to a large scale, here the modes to be outside the horizon.

The curvature perturbation in terms of a Fourier series can be written as

$$
\mathcal{R}=\int \frac{d^{3} \boldsymbol{k}}{(2 \pi)^{3 / 2}} \mathcal{R}_{\boldsymbol{k}}(\eta) e^{i \boldsymbol{k} \boldsymbol{x}},
$$

and the vacuum expectation value is defined as $\left\langle\mathcal{R}_{\boldsymbol{k}} \mathcal{R}_{l}^{*}\right\rangle=\frac{2 \pi}{k^{3}} \mathcal{P}_{R} \delta^{3}(\boldsymbol{k}-\boldsymbol{l})$, where $\mathcal{P}_{R}(k)$ is the power spectrum. In this form, the power spectrum of the curvature perturbations is given by

$$
\mathcal{P}_{R}(k)=\frac{k^{3}}{2 \pi^{2}}\left|\frac{u_{k}}{z}\right|^{2} .
$$

Now considering the power spectrum of the curvature perturbations the scalar index $n_{s}$ is defined as

$$
n_{s}-1=\frac{d \ln \mathcal{P}_{R}}{d \ln k} .
$$


In this way, we note that the evolution of scalar perturbations during the inflationary scenario is determined by the function $z(\eta)$, but this function $z(\eta)$ at the same time is determined through of the effective potential $U(\eta)=z^{\prime \prime} / z$. In this form, instead of considering a specific function $z(\eta)$, we can obtain exact solutions from Eqs. (13) and (14), considering the effective potential $U(\eta)$ itself, see e.g. [20].

On the other hand, the production of tensor perturbation during the inflationary epoch would generate gravitational waves. The formalism to study the quantum fluctuations in the gravitational field is similar to the case of the scalar perturbation discussed above. Here, the equation for the Fourier modes $v_{k}$, from the effective gravitational action is given by [24, 25]

$$
v_{k}^{\prime \prime}+\left(k^{2}-\frac{a^{\prime \prime}}{a}\right) v_{k}=0,
$$

and the power spectrum of the tensor perturbations in terms of the modes $v_{k}$, is defined as

$$
\mathcal{P}_{g}=\frac{2 k^{3}}{\pi^{2}}\left|\frac{v_{k}}{a}\right|^{2},
$$

where now the tensor spectral index $n_{T}$, is given by $n_{T}=d \ln \mathcal{P}_{g} / d \ln k$.

For the case of the tachyonic field, the equation for the Fourier modes $v_{k}$ is exactly the same as in the standard scalar field Eq.(18), because in absence of anisotropic stress gravity waves are decoupled from matter. Also, an important observational quantity is the tensor to scalar ratio $r$, which is defined as

$$
r=\frac{\mathcal{P}_{g}}{\mathcal{P}_{R}} .
$$

The Planck collaboration published new data for the ratio $r$, and found an upper bound

on the tensor to scalar ratio in which $r_{0.002}<0.11$ (95\% C.L.) [5], and this upper bound for the tensor to scalar ratio is similar to obtained from the Planck mission in which $r<0.12$ (95\% C.L.) [29].

In the following we will study different forms of the effective potential of cosmological perturbations $U(\eta)$, in the context of the Born-Infeld theory.

\section{EASTHER'S MODEL: TACHYON FIELD AND EXACT SOLUTIONS}

In this section we will describe the Easther's model in the context of a tachyonic field. In this case, which is the simplest case in which the function $z(\eta)=$ constant or equivalently the effective potential $U(\eta)=0$. In particular for the standard scalar field or originally the 
Easther's model, the differential equation for the Hubble parameter from the equation of the Fourier modes is given by, see Ref.[19]

$$
\frac{1}{2}+\left(\frac{H_{, \phi}}{H}\right)^{2}-\frac{H_{, \phi \phi}}{H}=0
$$

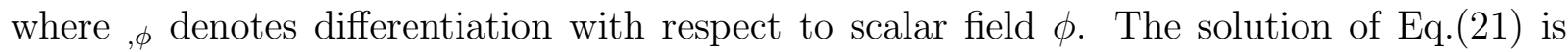
given by $H(\phi)=B \exp \left[\phi^{2} / 4+A \phi\right]$, in which $A$ and $B$ are two integration constants. In this case the spectrum of density perturbations becomes $\mathcal{P}_{R}(k) \propto k^{2}$, and then the exact spectral index $n_{s}=3$. In this form, for a standard scalar field, the cosmological quantities are exact, and in particular the spectral index becomes independent of the scalar field. However, this value of the spectral index $n_{s}$ is disapproved from observational data.

For the tachyonic field the Eq.(14) can be modified by defining a new variable $\Theta_{k}=u_{k} / z$, such that

$$
\Theta_{k}^{\prime \prime}+\frac{2}{z} z^{\prime} \Theta_{k}^{\prime}+k^{2}\left(1-\dot{\varphi}^{2}\right) \Theta_{k}=0
$$

Considering that the variable $z$ for the tachyon field is given by Eq.(13), then the Eq.(22) can be rewritten as

$$
\Theta_{k}^{\prime \prime}+\frac{4}{3} \frac{a}{H^{2}}\left(H^{4}-\frac{4}{9} H_{, \varphi}^{2}\right)^{-1}\left[\frac{H_{, \varphi \varphi}}{H}-\frac{4}{3}\left(\frac{H_{, \varphi}}{H}\right)^{2}-\frac{3}{2} H^{2}\right] \Theta_{k}^{\prime}+k^{2}\left(1-\dot{\varphi}^{2}\right) \Theta_{k}=0 .
$$

Assuming that the function $z(\eta)$ is a constant, then from Eq.(23) the square brackets is equal to zero in which

$$
\frac{H_{, \varphi \varphi}}{H}-\frac{4}{3}\left(\frac{H_{, \varphi}}{H}\right)^{2}-\frac{3}{2} H^{2}=0,
$$

and the solution of Eq.(24) can be written as

$$
H(\varphi)=\Im^{-1}[\varphi]
$$

where the function $\Im^{-1}[\varphi]$ corresponds to the inverse function of $\Im[\varphi]$ defined as

$\Im[\varphi]=\frac{6\left(4 C_{1}\left(\varphi+C_{2}\right)+9\left(\varphi+C_{2}\right)^{7 / 3}\right)-9\left(\varphi+C_{2}\right)^{7 / 3} \sqrt{4+\frac{9\left(\varphi+C_{2}\right)^{4 / 3}}{C_{1}}}{ }_{2} F_{1}\left[\frac{1}{2}, \frac{3}{4}, \frac{7}{4},-\frac{9\left(\varphi+C_{2}\right)^{4 / 3}}{4 C_{1}}\right]}{4 C_{1} \sqrt{4 C_{1}\left(\varphi+C_{2}\right)^{8 / 3}+9\left(\varphi+C_{2}\right)^{4}}}$,

in which ${ }_{2} F_{1}$ is the hypergeometric function. The constants $C_{1}$ and $C_{2}$ are two integration constants. Note the difference between the Eqs.(21) and (24) for the Easther's model, in which the quantity $z=$ const.. 
From Eq.(8) the effective tachyonic potential is given by

$$
V(\varphi)=\frac{3 H^{2}}{\kappa} \sqrt{1-\frac{4}{9}\left(\frac{H_{, \varphi}}{H^{2}}\right)^{2}}=\frac{3 \Im^{-1}[\varphi]^{2}}{\kappa} \sqrt{1-\frac{4}{9}\left(\frac{\Im^{-1}[\varphi]_{, \varphi}}{\Im^{-1}[\varphi]^{2}}\right)^{2}},
$$

here we have considered the exact solution $H=H(\varphi)$ given by Eq.(25).

For the tachyonic field the inflationary scenario occurs when

$$
\dot{\varphi}^{2}=\frac{4}{9} \frac{H_{, \varphi}^{2}}{H^{4}}=\frac{4}{9}\left(\frac{\Im^{-1}[\varphi]_{, \varphi}}{\Im^{-1}[\varphi]^{2}}\right)^{2}<\frac{2}{3} .
$$

Here we have considered Eq.(7) in which $\dot{\varphi}=-\frac{2}{3} \frac{H_{, \varphi}}{H^{2}}$.

In Fig1, we show the evolution of the effective tachyonic potential $V(\varphi)$ versus the tachyon field $\varphi$. Here, we considered two different values of the integration constant $C_{1}$. In particular, the solid line corresponds to $C_{1}=0.1$ and $C_{2}=1$, and the dashed line is for the case in which $C_{1}=0.2$ and $C_{2}=1$.

In order to write down the quantity that relates $V(\varphi)$, with the tachyonic field $\varphi$, we consider Eq.(26), and we use the values $C_{2}=1$ and $m_{p}=1$. From this plot we note that the effective tachyonic potential $V(\varphi)$ satisfies the properties: $V_{, \varphi}(\varphi>0)<0$ and $V(\varphi \rightarrow \infty) \rightarrow 0$, that are the essential conditions for a tachyonic effective potential.

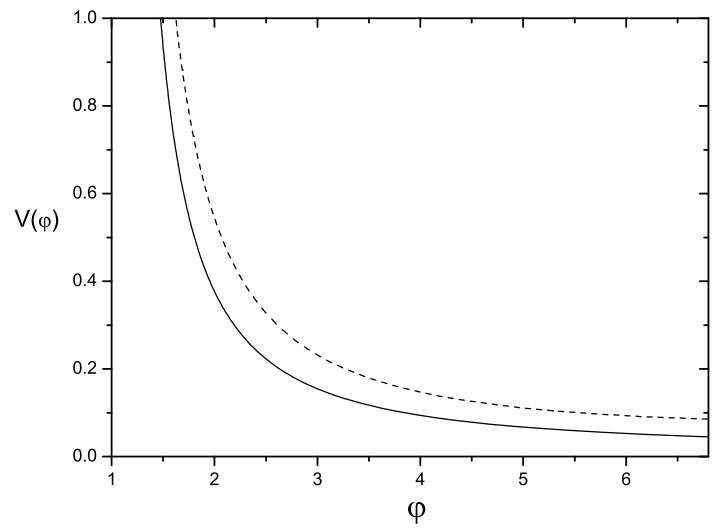

FIG. 1: This plot shows the dependence of the effective tachyonic potential $V(\varphi)$ versus the tachyonic field $\varphi$. Here, we considered two different values of the constant $C_{1}$. The solid and dashed lines correspond to $C_{1}=0.1$ and $C_{1}=0.2$, respectively. In this plot we have used the values $C_{2}=1$ and $m_{p}=1$. 


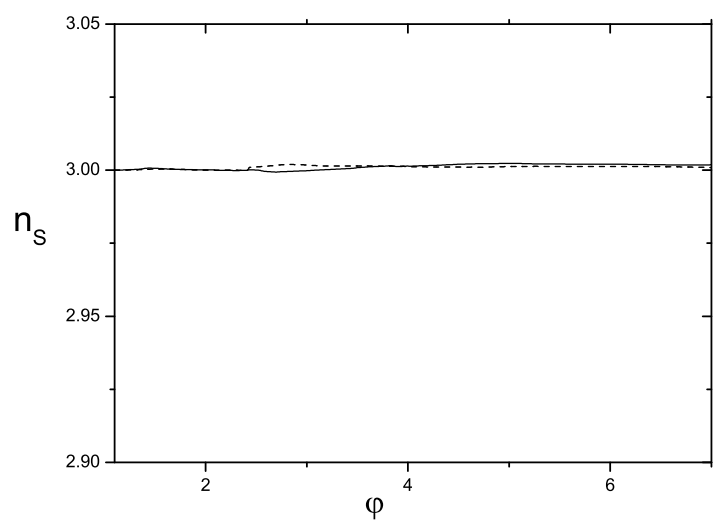

FIG. 2: Evolution of the spectral index $n_{s}$ versus the tachyonic field $\varphi$. As before, the solid and dashed lines correspond to $C_{1}=0.1$ and $C_{1}=0.2$, respectively. In this plot we have considered the values $C_{2}=1$ and $m_{p}=1$

Although all the quantities from the background field equations can be found analytically for the case in which the variable $z=$ constant, it is not possible to determine the corresponding cosmological perturbations analytically, since we need to use Eq.(27) together Eq.(14) with $U=0$. In Fig, 2 we show the spectral index $n_{s}$ versus the tachyonic field $\varphi$ for the case in which $z=$ constant. In order to write down the spectral index $n_{s}$, with the tachyonic field $\varphi$, firstly we consider Eqs.(14) in which $U=0$ (since the variable $z=$ constant) together with the exact solution given by Eq.(27). In this way, we numerically find the Fourier modes $u_{k}$, and therefore the power spectrum of the curvature perturbations for $z=z_{0}=$ const.. Now, considering Eq.(17) we numerically obtain the spectral index in terms of the tachyon field. From Fig,2 we observe that the spectral index is $n_{s} \simeq 3$, but this value is disfavored from observational data. Here, we observe that this results is very similar to obtained for the standard scalar field, in which $n_{S} \equiv 3$ (exact result), see Ref.[19]. This numerical result for the spectral index in the case of the tachyonic field is not a surprise, since if we consider that the tachyon field slowly changes with the cosmic time, then the effective speed of sound $c_{S}^{2} \sim\left(1-\dot{\varphi}_{0}^{2}\right)=c_{S_{0}}^{2}=$ const., and the solution of Eq.(14) for $z=$ const., is given by $u_{k}(\eta)=(1 / \sqrt{k}) e^{-i c_{S_{0}} k}$, wherewith $n_{s} \equiv 3$. 


\section{ANSATZ: EFFECTIVE POTENTIAL $U(\eta)$}

In this section we will study a specific ansatz for the effective potential $U(\eta)$ of cosmological perturbations. Following Ref.[20], a possible ansatz for the effective potential is given by $U(\eta)=\sum_{m=1}^{M} \frac{C_{m}}{|\eta|^{m}}$, where the constants $C_{m}$ 's should be calculated for different inflationary model. For instance in the case in which the constants $C_{m}=0$ if $m \neq 2$, represents to power-law inflation together with the inflationary models in the slow-roll approximation[20], and de Sitter inflation occurs when $C_{2}$ is exactly $C_{2} \equiv 2$.

In this form, considering exact results from the effective potential of cosmological perturbations; power law inflation $\left(a \sim t^{p}\right.$ with $\left.p>1\right)$ in which $U(\eta)=\frac{\nu^{2}-1 / 4}{\eta^{2}}$, where the constant

$\nu$ is given by $\nu=3 / 2+1 /(p-1)[18]$, and for de Sitter inflation $U(\eta)=\frac{2}{\eta^{2}}[24]$, we consider the effective potential of cosmological perturbations can be written as

$$
U(\eta)=\frac{z^{\prime \prime}}{z}=C_{0}+\frac{C_{1}}{\eta}+\frac{C_{2}}{\eta^{2}}
$$

where $C_{0}, C_{1}$ and $C_{2}$ are free parameters. Here the parameter $C_{0}$ has dimensions of masssquared, $C_{1}$ of mass and $C_{2}$ is dimensionless.

Considering the change of the variable $\bar{\eta}=2 \sqrt{C_{0}} \eta$, then Eq.(28) can be rewritten as

$$
\frac{d^{2} z}{d \bar{\eta}^{2}}+\left(-\frac{1}{4}+\frac{\alpha}{\bar{\eta}}+\frac{\frac{1}{4}-\beta^{2}}{\bar{\eta}^{2}}\right) z(\bar{\eta})=0,
$$

where the quantities $\alpha$ and $\beta$ are constants, and are defined as

$$
\alpha=\frac{-C_{1}}{2 \sqrt{C_{0}}} \text {, and } \beta^{2}=\frac{1}{4}+C_{2},
$$

respectively. The exact solution of Eq.(29) is given in terms of Whittaker functions $W_{ \pm \alpha, \beta}$ [30], and the solution for the variable $z$ can be written as

$$
z(\bar{\eta})=A_{1} W_{\alpha, \beta}(\bar{\eta})+A_{2} W_{-\alpha, \beta}(-\bar{\eta})
$$

where $A_{1}$ and $A_{2}$ are two integration constants. We note that in general our variable fundamental $z$ is characterized by the constants $C_{0}, C_{1}$ and $C_{2}$, and two integration constants $A_{1}$ and $A_{2}$.

In order to obtain analytical solutions for the background field equations and the cosmological perturbations (scalar and tensor perturbations), we considered that the tachyonic field slowly changes with the cosmic time, and the velocity $\dot{\varphi}$ is approximately constant, i.e., 
$\dot{\varphi}^{2} \sim \dot{\varphi}_{0}^{2}=$ const. $<2 / 3$, then the effective speed of sound becomes $c_{S}^{2} \sim\left(1-\dot{\varphi}_{0}{ }^{2}\right)=c_{S_{0}}^{2}$, where $c_{S_{0}}^{2}>1 / 3$.

Under this approximation, and considering the Eqs.(13) and (31), we find that the scale factor $a$ is given by $a(\eta)=\bar{A}_{1} W_{\alpha, \beta}\left(2 \sqrt{C_{0}} \eta\right)$, where the constant $\bar{A}_{1}$ is defined as $\bar{A}_{1}=$ $c_{S_{0}} A_{1} / \sqrt{3\left(1-c_{S_{0}}^{2}\right)}$, and from Eq.(8) the tachyonic potential in terms of the conformal time $\eta$ can be written as

$$
V(\eta)=\frac{3}{\kappa} \frac{\mathcal{H}^{2}}{a^{2}} \sqrt{\frac{1}{3}\left[1+\frac{2 \mathcal{H}^{\prime}}{\mathcal{H}^{2}}\right]}=\frac{3}{\kappa \bar{A}_{1}^{2}}\left[\frac{W_{\alpha, \beta}^{\prime}}{W_{\alpha, \beta}^{2}}\right]^{2} \sqrt{\frac{1}{3}\left[1+\frac{2\left(W_{\alpha, \beta} W_{\alpha, \beta}^{\prime \prime}-W_{\alpha, \beta}^{\prime 2}\right)}{W_{\alpha, \beta}^{\prime 2}}\right]},
$$

where $\mathcal{H}=H a=a^{\prime} / a$, and $W_{\alpha, \beta}$ corresponds to the Wittaker function $W_{\alpha, \beta}\left(2 \sqrt{C_{0}} \eta\right)$. Here we have used that the constant $A_{2}=0$ (without loss of generality), and as before a prime denotes differentiation with respect the conformal time $\eta$. Considering the $\dot{\varphi}=$ $\varphi^{\prime} / a \sim \dot{\varphi}_{0}=\left(1-c_{S_{0}}\right)^{1 / 2}$, we obtain that $\varphi(\eta) \propto \int W_{\alpha, \beta} d \eta$, and then we would obtain $V=V(\varphi)$. However, the field $\varphi(\eta)$ does not have an analytical solution. Instead of consider this numerical integral, we numerically solve the full background equations.

In Fig3, we show the evolution of the effective tachyonic potential $V(\varphi)$ versus the tachyon field $\varphi$. In doing this, we considered two different values of the constant $C_{1}$. Here, the doted line corresponds to $C_{1}=10^{-5}$ and solid line to $C_{1}=10^{-7}$. Also, in this plot we have used the values $C_{0}=10^{-8}, C_{2}=2, A_{1}=10^{-6}$ and $m_{p}=1$. In order to write down the effective tachyonic potential $V(\varphi)$ versus $\varphi$, we numerically utilize Eqs.(13) and (28), together with the background equations given by Eqs.(4)-(6). From this plot, we observe that the tachyonic effective potential $V(\varphi)$ satisfies the properties; $V_{, \varphi}(\varphi>0)<0$ and $V(\varphi \rightarrow \infty) \rightarrow 0$, that are the conditions for an effective tachyonic potential. We note that this tachyonic potential is analogous to obtained in the Easther's model (see section IV).

Now from Eqs.(14) and (28), the equation for the Fourier modes $u_{k}$, becomes

$$
u_{k}^{\prime \prime}(\eta)+\left(c_{S_{0}}^{2} k^{2}-C_{0}-\frac{C_{1}}{\eta}-\frac{C_{2}}{\eta^{2}}\right) u_{k}(\eta)=0 .
$$

Redefining the conformal time as $\tilde{\eta}=2 i \sqrt{c_{S_{0}}^{2} k^{2}-C_{0}} \eta$, the above equation results

$$
\frac{d^{2} u_{k}}{d \tilde{\eta}^{2}}+\left(-\frac{1}{4}+\frac{\tilde{\alpha}}{\tilde{\eta}}+\frac{\frac{1}{4}-\beta^{2}}{\tilde{\eta}^{2}}\right) u_{k}=0
$$

where now the constant $\tilde{\alpha}$ is defined by $\tilde{\alpha}=\frac{C_{1} i}{2 \sqrt{c_{S_{0}}^{2} k^{2}-C_{0}}}$, and the constant $\beta$ as before is given by Eq.(30). From Eq.(34), we find that the analytical solution for the Fourier modes 


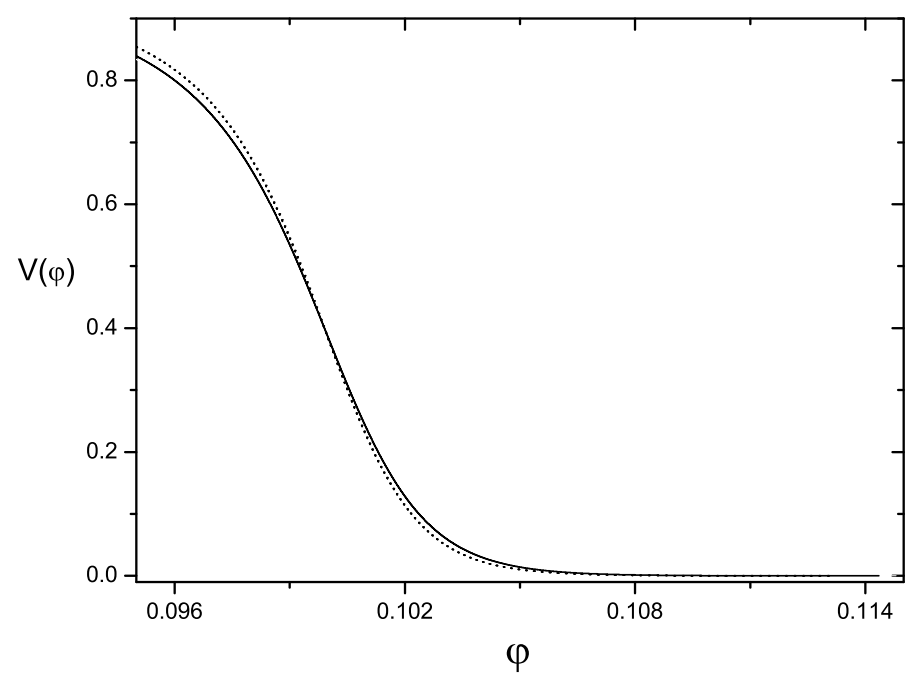

FIG. 3: Numerical result: Evolution of the effective potential $V(\varphi)$ versus the tachyonic field $\varphi$. Here, the doted and solid lines correspond to $C_{1}=10^{-5}$ and $C_{1}=10^{-7}$, respectively. In this plot we have considered the values $C_{0}=10^{-8}, C_{2}=2, A_{1}=10^{-6}$ and $m_{p}=1$.

can be written as

$$
u_{k}(\tilde{\eta})=B_{1} W_{\tilde{\alpha}, \beta}(\tilde{\eta})+B_{2} W_{-\tilde{\alpha}, \beta}(-\tilde{\eta})
$$

where $B_{1}$ and $B_{2}$ are two integration constants, and whose values are fixed by the initial conditions, and as before $W_{ \pm \tilde{\alpha}, \beta}$ correspond to the Whittaker functions.

In order to fix the constants $B_{1}$ and $B_{2}$, we consider the small scale limit in which the conformal time $\eta \rightarrow-\infty$, then from Eq.(35) we get

$$
u_{k}(\eta) \rightarrow \frac{1}{\sqrt{2 k_{e f f}}} e^{-i k_{e f f} \eta},
$$

where $k_{\text {eff }}$ corresponds to the effective modulus of wavenumber, and is given by $k_{\text {eff }}=$ $\sqrt{c_{S_{0}}^{2} k^{2}-C_{0}}>0$. Here, we observe that $k_{e f f}$ represents a shift in the effective wavenumber $c_{S_{0}} k$. Now considering that in the asymptotic limit $\eta \rightarrow-\infty$ (small scale limit) the Whittaker function is proportional to $e^{-\frac{\tilde{\eta}}{2}} \tilde{\eta}^{\alpha}$, we get

$$
B_{1}=\frac{e^{\frac{\pi C_{1}}{4 k_{e f f}}}}{\sqrt{2 k_{e f f}}} \text {, and } B_{2}=0 .
$$

Here, we have used the Wronskian condition. By using the Eqs.(35) and (36), we find that the analytical solution for the Fourier modes can be written as 


$$
u_{k}(\tilde{\eta})=\frac{e^{\frac{\pi C_{1}}{4 k_{e f f}}}}{\sqrt{2 k_{e f f}}} W_{\tilde{\alpha}, \beta}(\tilde{\eta})
$$

On the other hand, we consider the growing modes in the large scale limit in which the conformal time $\eta \rightarrow 0$ (the quantum fluctuations are frozen outside the horizon) the Whittaker function becomes $W_{\tilde{\alpha}, \beta}(\tilde{\eta}) \rightarrow \frac{\Gamma(2 \beta)}{\Gamma\left(\frac{1}{2}+\beta-\tilde{\alpha}\right)} \tilde{\eta}^{1 / 2-\beta} e^{-\frac{\tilde{\eta}}{2}}$, and then we obtain that the analytical solution for $u_{k}(\eta)$ for large scale can be written as

$$
u_{k}(\eta)=\frac{e^{\pi C_{1} / 4 k_{e f f}}}{\sqrt{2 k_{e f f}}} \frac{\Gamma(2 \beta)}{\Gamma\left(\frac{1}{2}+\beta-\tilde{\alpha}\right)}\left(2 i k_{e f f} \eta\right)^{1 / 2-\beta} e^{-i k_{e f f} \eta}
$$

In this form, considering Eqs.(16) and (38), we find that the power spectrum of the curvature perturbations becomes

$$
P_{R}(k)=B \frac{k^{3} k_{e f f}^{-2 \beta}}{\Gamma(s) \Gamma^{*}(s)} e^{\pi C_{1} / 2 k_{e f f}}, \text { where } B=\frac{\Gamma^{2}(1 / 2+\beta-\alpha)}{4 \pi^{2} A_{1}^{2} C_{0}^{1 / 2-\beta}} \text {, and } s=\frac{1}{2}+\beta-\tilde{\alpha} \text {. }
$$

Here, we considered that in the limit $\bar{\eta} \rightarrow 0$ (large scale limit), the variable $z$ is given by $z(\bar{\eta}) \rightarrow A_{1} \frac{\Gamma(2 \beta)}{\Gamma\left(\frac{1}{2}+\beta-\alpha\right)} \bar{\eta}^{\frac{1}{2}-\beta}$, and as before the constant $A_{2}=0$.

From the power spectrum given by Eq.(39), we find that the spectral scalar index $n_{s}$, considering Eq.(17) is given by

$$
n_{s}(k)=4-\frac{c_{s 0}^{2} k^{2}}{k_{e f f}}\left[2 \beta k_{e f f}^{2 \beta}+\frac{C_{1}}{2 k_{e f f}^{2}}\left(\pi+i\left[\Psi_{0}(s)-\Psi_{0}\left(s^{*}\right)\right]\right)\right],
$$

where $\Psi_{0}(s)$ corresponds to the Polygamma function.

In the context of the spectrum of gravity waves for a tachyonic field, the equation of the Fourier modes $v_{k}$ is exactly the same as in the standard scalar field, see Eq.(18). In this form, by considering Eqs. (13) and (18), the equation of the Fourier modes $v_{k}$ can be written as

$$
\frac{d^{2} v_{k}}{d \eta_{T}^{2}}+\left(-\frac{1}{4}+\frac{\alpha_{T}}{\eta_{T}}-\frac{\frac{1}{4}-\beta^{2}}{\eta_{T}^{2}}\right) v_{k}=0
$$

where the constant $\alpha_{T}=\frac{C_{1} i}{2 \sqrt{k^{2}-C_{0}}}$, the constant $\beta$ as before is given by Eq.(30), and the new conformal time is defined as $\eta_{T}=2 i \sqrt{k^{2}-C_{0}} \eta$. In this way, we obtain that the exact solution for the modes $v_{k}$ of Eq.(41) becomes

$$
v_{k}(\eta)=D_{1} W_{\alpha_{T}, \beta}\left(2 i \sqrt{k^{2}-C_{0}} \eta\right)+D_{2} W_{-\alpha_{T}, \beta}\left(-2 i \sqrt{k^{2}-C_{0}} \eta\right)
$$

where $D_{1}$ and $D_{2}$ are two integration constants. In particular for the Sitter universe, the scale factor is $a \propto-\eta^{-1}$, and the equation of the modes $v_{k}$ is $v_{k}^{\prime \prime}+\left(k^{2}-\frac{2}{\eta^{2}}\right) v_{k}=0$, that in 
our case corresponds to $C_{0}=0, C_{1}=0$ and $C_{2}=2$, and the solution for the modes $v_{k}$, is given by $v_{k}=(1 / \eta)\left[D_{1}(k \eta \cos [k \eta]-\sin [k \eta])+D_{2}(k \eta \sin [k \eta]+\cos [k \eta])\right]$, see Ref.[24].

Following Ref. [24], the initial conditions for the modes $v_{k}\left(\eta_{i}\right)$ and $v_{k}^{\prime}\left(\eta_{i}\right)$ (initial conformal time $\eta_{i}$ ) are given by

$$
v_{k}\left(\eta_{i}\right)=\frac{1}{\left[k^{2}-C_{0}-\frac{C_{1}}{\eta_{i}}-\frac{C_{2}}{\eta_{i}^{2}}\right]^{1 / 4}}, \quad \text { and } v_{k}^{\prime}\left(\eta_{i}\right)=i\left[k^{2}-C_{0}-\frac{C_{1}}{\eta_{i}}-\frac{C_{2}}{\eta_{i}^{2}}\right]^{1 / 4},
$$

respectively. Here, the initial conditions make sense exclusively if $\left[k^{2}-C_{0}-\frac{C_{1}}{\eta_{i}}-\frac{C_{2}}{\eta_{i}^{2}}\right]>0$. Considering the solution of the modes $v_{k}$, together the initial conditions given by Eq.(43), we find that the constants $D_{1}$ and $D_{2}$ becomes

$D_{1}=\frac{\alpha_{T}}{2 \sqrt{k_{B}}} \frac{\left(2 k_{B} \eta_{i}\right)^{-\frac{i C_{1}}{2 k_{B}}}}{\left(\alpha_{T}+i k_{B} \eta_{i}\right)} e^{i k_{B} \eta_{i}+\frac{\pi C_{1}}{4 k_{B}}}$, and $D_{2}=\frac{1}{2 \sqrt{k_{B}}} \frac{\left(\alpha_{T}+2 i k_{B} \eta_{i}\right)\left(-2 k_{B} \eta_{i}\right)^{\frac{i C_{1}}{2 k_{B}}}}{\left(\alpha_{T}+i k_{B} \eta_{i}\right)} e^{-\left(i k_{B} \eta_{i}+\frac{\pi C_{1}}{4 k_{B}}\right)}$.

Here, we considered that for $\eta \longrightarrow-\infty$ (small scale limit), the normalization given by Eq.(43) becomes $\left[k^{2}-C_{0}-\frac{C_{1}}{\eta_{i}}-\frac{C_{2}}{\eta_{i}^{2}}\right]^{1 / 4} \simeq\left[k^{2}-C_{0}\right]^{1 / 4}=\sqrt{k_{B}}>0$, where $k_{B}=\sqrt{k^{2}-C_{0}}$, and also we assumed that the asymptotic behavior of the Wittaker function in the small scale limit is $W_{\alpha_{T}, \beta}\left(2 i k_{B} \eta\right) \rightarrow\left(2 i k_{B} \eta\right)^{\alpha_{T}} e^{-i k_{B} \eta}$. Also, we note that in particular for the case in which $C_{0}=0$, the initial conditions are given by $v_{k}\left(\eta_{i}\right)=1 / \sqrt{k}$ and $v_{k}^{\prime}\left(\eta_{i}\right)=i \sqrt{k}$, when $\eta \longrightarrow-\infty[24]$.

In this form, from Eqs.(31) and (42), the power spectrum of the tensor perturbation, is given by

$$
\mathcal{P}_{g}=\frac{2 k^{3}}{\pi^{2}}\left|\frac{v_{k}}{a}\right|^{2}=\frac{2 k^{3}}{\pi^{2} \tilde{A}_{1}^{2}} \frac{\left|D_{1} W_{\alpha_{T}, \beta}\left(2 i k_{B} \eta\right)+D_{2} W_{-\alpha_{T}, \beta}\left(-2 i k_{B} \eta\right)\right|^{2}}{\left|W_{\alpha, \beta}\left(2 \sqrt{C_{0}} \eta\right)\right|^{2}}
$$

where the constant $\tilde{A}_{1}$ is defined as $\tilde{A}_{1}=\frac{c_{S_{0}} A_{1}}{\sqrt{3\left(1-c_{S_{0}}^{2}\right)}}$.

Considering the large scale limit in which $\eta \rightarrow 0$, then the spectrum of the gravitational waves given by Eq.(44), can be written as

$$
\begin{gathered}
\mathcal{P}_{g}(k) \simeq \frac{2 k^{3}}{\pi^{2} \tilde{A}_{1}{ }^{2}}|\Gamma(1 / 2+\beta-\alpha)|^{2}\left(\frac{k_{B}^{2}}{C_{0}}\right)^{1 / 2-\beta} \times\left[\frac{\left|D_{1}\right|^{2}}{\left|\Gamma\left(1 / 2+\beta-\alpha_{T}\right)\right|^{2}}\right. \\
\left.+\frac{\left|D_{2}\right|^{2}}{\left|\Gamma\left(1 / 2+\beta+\alpha_{T}\right)\right|^{2}}+(-1)^{1 / 2-\beta} \operatorname{Re}\left(\frac{2 D_{1} D_{2}^{*}}{\Gamma\left(1 / 2+\beta-\alpha_{T}\right) \Gamma^{*}\left(1 / 2+\beta+\alpha_{T}\right)}\right)\right] .
\end{gathered}
$$

Here, we considered that in the large scale limit $(\eta \rightarrow 0)$, the Wittaker function is given by $W_{\alpha_{T}, \beta} \rightarrow\left[\Gamma(2 \beta) / \Gamma\left(1 / 2+\beta-\alpha_{T}\right)\right]\left(2 i k_{B} \eta\right)^{1 / 2-\beta} e^{-i k_{B} \eta}$. 


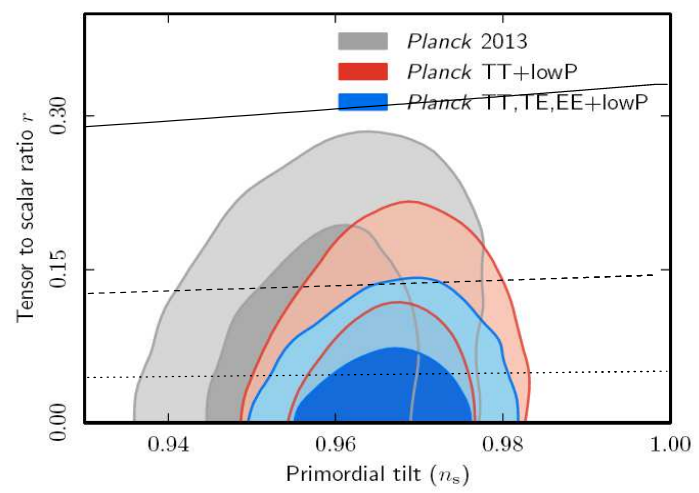

FIG. 4: The plot shows the tensor to scalar ratio $r$ versus the scalar spectrum index $n_{s}$ in the case of the tachyonic field $\varphi$, for different values of the speed of sound $c_{S_{0}}$. In this plot we show the two-dimensional marginalized constraints (68\% and $95 \%$ C.L.) on the parameters $r$ and $n_{s}$ obtained from the Planck 2015 data [5]. Here, the solid, dashed, and doted lines correspond to $c_{S_{0}}=0.993, c_{S_{0}}=0.997$ and $c_{S_{0}}=0.999$, respectively. In this plot we have considered the values $\eta_{i}=-400.000, C_{0}=10^{-8}, C_{1}=1, C_{2}=2$ and $m_{p}=1$.

In Fig 4 we show the tensor to scalar ratio $r$ versus the scalar spectral index $n_{s}$, for the effective potential of cosmological perturbations $U(\eta)$ given by the ansantz of Eq.(28). Here, we show the two-dimensional marginalized constraints for the tensor to scalar ratio $r$ versus $n_{s}$, from the new results of Planck 2015 data[5]. Combining Eqs.(39), (40) and (45), we numerically find the consistency relation $r=r\left(n_{s}\right)$, and also we consider three values of the effective speed of sound. The solid, dashed, and doted lines correspond to the speed sound $c_{S_{0}}=0.993, c_{S_{0}}=0.997$ and $c_{S_{0}}=0.999$, respectively. Also, in this plot we have used the values $\eta_{i}=-400.000, C_{0}=10^{-8}, C_{1}=1, C_{2}=2$ and $m_{p}=1$. From the two-dimensional marginalized constraints we note that for the value $c_{S_{0}}>0.993$ the model is well supported by the new Planck data. In this form, we observe that for the case of de Sitter inflation in which $C_{2}=2$ (recalled that in de Sitter $U(\eta)=2 / \eta^{2}$ ), and for different values of $C_{0}$ (with $c_{S_{0}}^{2} k^{2}>C_{0}$ ) and $C_{1}<10^{2}$, the tachyonic model is very similar to the standard scalar field, where $c_{S} \equiv 1$. Therefore, it may come as a surprise that exist a similitude in the trajectories $n_{s}-r$ plane between standard field and tachyonic field. However, this similitude was noted in 
Ref.[14] at lower order in the slow roll parameters. Also, we observe that for small deviations of the value of $C_{2}=2$ ( for instance, power law inflation $U(\eta)=\left(\nu^{2}-1 / 4\right) / \eta^{2}$ ), we find that for values of $C_{2}<2-0.1$ the model is disfavored from observational data, since the spectral index $n_{s}>1$, and for values of $C_{2}>2+0.1$ the ratio $r>0.2$, also the model is disfavored from observational data. In this form, for deviations of the value of $C_{2}=2$ (de Sitter inflation) our model does not work.

\section{CONCLUSIONS}

In this paper we have analyzed an approach to exact solutions of cosmological perturbations in the context of the Born-Infeld theory for two models; the Easther's model, and the ansatz $U(\eta)$ given by Eq.(28). For both models, we have found explicit solutions for the corresponding scalar field, effective tachyonic potential, power spectrum of the curvature perturbations $P_{R}$, scalar spectrum index $n_{s}$, power spectrum of the tensor perturbations $\mathcal{P}_{g}$ and the tensor to scalar ratio $r$.

For the Easther's model in which $U=0$, we have obtained that only the quantities from the background field equations can be found analytically. In particular, we have got an effective tachyonic potential with the properties $V_{, \varphi}(\varphi>0)<0$ and $V(\varphi \rightarrow \infty) \rightarrow 0$. However, we have found that numerically the spectral index $n_{s} \simeq 3$, but this value is disfavored from observational data. This result for the spectral index is of according with the numerical solution of $n_{s}=n_{s}(\varphi)$, if we assumed that the tachyon field slowly changes with the cosmic time, and then $n_{s} \equiv 3$.

For the model in which the effective potential of cosmological perturbations is given by $U(\eta)=C_{0}+C_{1} / \eta+C_{2} / \eta^{2}$, we have found analytical results assuming that the tachyon field $\varphi$ slowly changes with the time. Under this approximation we have obtained that the scalar and tensor perturbations can be written in terms of the Wittaker functions and essentially from definitions of $U(\eta)$ and $z$ for the tachyonic field, see Eq.(13). Considering the asymptotic limits for each one of the Fourier modes (scalar and tensor modes) we have obtained the power spectrums scalar and tensorial in terms of the modulus of the wavenumber $k$. From these results we have considered the constraints on the parameters in our model from the Planck 2015 data. Here we have taken the constraint $r=r\left(n_{s}\right)$ plane or consistency relation, and we have observed that for deviations of the value $C_{2}=2$ (de Sitter inflation) our model 
is disfavored from observational data, and then the model does not work. Also, we have noted from the trajectories $n_{s}-r$ plane, that our model is well supported by the Planck data only if the effective speed of sound $c_{S_{0}}>0.993$ (see Fig, (4) that is very similar to the speed of sound of the standard scalar field $\left(c_{S} \equiv 1\right)$. Therefore, our ansatz of the effective potential of cosmological perturbations, the tachyonic model works for values of $c_{S_{0}} \simeq 1$ and $C_{2} \simeq 2$

\section{Acknowledgments}

R.H. was supported by Comisión Nacional de Ciencias y Tecnología of Chile through FONDECYT Grant N $N^{0} 1130628$ and DI-PUCV N ${ }^{0}$ 123.724. R.G.P was supported by Proyecto Beca-Doctoral CONICYT N² 21100145

[1] A. Guth, Phys. Rev. D 23, 347 (1981).

[2] A.A. Starobinsky, Phys. Lett. B 91, 99 (1980); A.D. Linde, Phys. Lett. B 108, 389 (1982).

[3] S. W. Hawking,Phys. Lett. B 115, 295 (1982);A. A. Starobinsky, Phys. Lett. B 117, 175 (1982); J.M. Bardeen, P.J. Steinhardt and M.S. Turner, Phys. Rev.D 28, 679 (1983).

[4] D. Larson et al., Astrophys. J. Suppl. 192, 16 (2011); C. L. Bennett et al., Astrophys. J. Suppl. 192, 17 (2011).

[5] P. A. R. Ade et al. [Planck Collaboration], arXiv:1502.02114 [astro-ph.CO].

[6] A. Sen, JHEP, 9910, 008, (1999).

[7] M. Fairbairn and M. H. Tytgat, Phys. Lett. B 546,1 (2002).

[8] L. Kofman and A. Linde, JHEP 0207, 004 (2002); M. Sami, P. Chingangbam and T. Qureshi, Phys. Rev. D 66, 043530 (2002).

[9] J. S. Bagla, H. K. Jassal and T. Padmanabhan, Phys. Rev. D 67, 063504 (2003); E. J. Copeland, M. R. Garousi, M. Sami and S. Tsujikawa, Phys. Rev. D 71, 043003 (2005).

[10] L. P. Chimento, Phys. Rev. D 69, 123517 (2004); R. Herrera, D. Pavon and W. Zimdahl, Gen. Rel. Grav. 36, 2161 (2004); I. Y. Aref'eva, AIP Conf. Proc. 826, 301 (2006); G. Calcagni and A. R. Liddle, Phys. Rev. D 74, 043528 (2006); M. R. Setare, Phys. Lett. B 653, 116 (2007).

[11] G. W. Gibbons, Phys. Lett. B 537, 1 (2002). 
[12] D. Kutasov, M. Marino and G. W. Moore, JHEP 0010, 045 (2000).

[13] A. A. Gerasimov and S. L. Shatashvili, JHEP 0010, 034 (2000); A. Sen, JHEP 0204, 048 (2002).

[14] D. A. Steer and F. Vernizzi, Phys. Rev. D 70, 043527 (2004).

[15] T. Padmanabhan, AIP Conf. Proc. 843, 111 (2006)

[16] V. Lukash, Pis'ma Zh. Eksp. Teor. Fiz. 49, 631 (1980); G. Chibisov and V. Mukhanov, Galaxy and phonons P.N. Lebedev Phys. Inst. 162 (1980); G. Chibisov and V. Mukhanov, Mon. Not. R. Astron. Soc. 200, 535 (1982).

[17] F. Lucchin, S. Matarrese, Phys. Rev. D 32, 1316 (1985).

[18] D. H. Lyth and E. D. Stewart, Phys. Lett. B 274, 168 (1992); E. D. Stewart and D. H. Lyth, Phys. Lett. B 302, 171 (1993).

[19] R. Easther, Class. Quantum Grav. 13, 1775 (1996).

[20] J. Martin, D. Schwarz, Phys.Lett.B 500, 1-7 (2001).

[21] I. J. Grivell and A. R. Liddle, Phys. Rev. D 54, 7191 (1996); A. Makarov, Phys. Rev. D 72, 083517 (2005).

[22] A. A. Starobinsky, JETP Lett. 82, 169 (2005); [Pisma Zh. Eksp. Teor. Fiz. 82, 187 (2005)].

[23] A. Sen, Mod. Phys. Lett.A 17,1797 (2002).

[24] V. F. Mukhanov, H. A. Feldman, and R. H. Brandenbarger, Phys. Rep. 215, 203 (1992).

[25] J. M. Bardeen, Phys. Rev. D 22, 1882 (1980); H. Kodama and M. Sasaki, Prog. Theor. Phys. Suppl. 78, 1 (1984); D. H. Lyth and A. Riotto, Phys. Rept. 314, 1 (1999).

[26] A. V. Frolov, L. Kofman and A. A. Starobinsky, Phys. Lett. B 545 (2002) 8.

[27] J. Garriga, V. F. Mukhanov, Phys. Lett. B458 , 219-225 (1999).

[28] J. c. Hwang and H. Noh, Phys. Rev. D 66, 084009 (2002).

[29] P. A. R. Ade et al. (BICEP2 Collaboration), Phys. Rev. Lett. 112, 241101 (2014); Astrophys. J. 792, 62 (2014).

[30] Abramowitz, M. and Stegun, I. A. (Eds.). Handbook of Mathematical Functions with Formulas, Graphs, and Mathematical Tables, 9th printing. New York: Dover, 1972; Arfken, G. "The Incomplete Gamma Function and Related Functions." Mathematical Methods for Physicists, 3rd ed. Orlando, FL: Academic Press, 1985. 